\title{
The design of detecting Aflatoxins based on continuous wave light source
}

\author{
Zhao Xuyang ${ }^{1, ~ a}$ Yang $\mathrm{Hao}^{1,}{ }^{\text {b }}$ Shen Guojin ${ }^{2,}$ Ding Yingjie ${ }^{2, d}$ \\ ${ }^{1}$ College of Information Engineering China Jiliang University Hangzhou 310000 \\ ${ }^{2}$ Yangtze Delta Region Institute of Tsinghua University Zhejiang Jiaxing 314000 \\ a11193122132@qq.com, 'b16317367@qq.com, 'c12991778@qq.com, d673554523@qq.com
}

\begin{abstract}
Keywords: closed loop feedback; stable light source; fixed wavelength; high frequency; Single Photon Counting
\end{abstract}

\begin{abstract}
. the separation of elements is achieved by ultra performance liquid chromatography system for Aflatoxins, the use of a closed-loop feedback control system is to produce a stable continuous wave light source, fixed wavelength of $360 \mathrm{~nm}$ illumination light source onto the separation of elements, resulting in the emission wavelength was $440 \mathrm{~nm}$ receiving photomultiplier tube, and then it gets high-frequency pulses. By single photon counting system pulse will be integrated for a fixed time, aflatoxin can be obtained G1, B1, G2, B2 content elements through the peak area calculation. Without derivatives quantitative determination of aflatoxin can be carried out quickly and efficiently. This design can effectively address the impact of the stability of the light source on the test results. It is useful for analysis LED spectral characteristics.
\end{abstract}

\section{Introduction}

Traditional aflatoxin detection methods are mainly thin layer chromatography,ultra performance liquid chromatography, immunochemical analysis. the presence of specificity is not strong, it is easy to interfere with other fluorescent substance, pre-treatment is more complicated. immunochemical analysis methods can only measured total aflatoxins [3]. in order to quickly detect food aflatoxin content, according to van Deemeter theoretical equation, with reducing particle size of LC system and increasing of chromatographic resolution,it turn out that the use of ultra-high performance liquid chromatography can be required to establish a small sample size, high degree of separation, fast and accurate reading of aflatoxin quantitative analysis methods [4].

\section{Aflatoxin detecting principle}

Single photon counting method for aflatoxin ingredients mainly used for determination of ultra performance liquid chromatography. using single photon counting mode has a characteristics of high integration, high precision, good stability. photon detector output discrete electrical characteristics under weak light illumination, and then the weak signal will be counted after extracting out in predetermined time, through data processing and curve drawing the elements of aflatoxin G1, B1, G2, B2 will be determined [5] .

Continuous wave light source is a major part of Chemiluminescence measurement system, this part emits a continuous wave light source of $370 \mathrm{~nm}$ fixed wavelength ultraviolet light, irradiation in the separation element will stimulate the reflected wave which is $440 \mathrm{~nm}$, reflected wave received by PMT photocathode will produce discrete pulse signal ,by the electric pulse signals the number of photons will be measured, the collected data is plotted, according to the peak area of aflatoxin test solution will be send to the PC software, after this processing the concentrate value will display $[6,7]$.

\section{Continuous wave light source design and analysis}

Aflatoxin testing needs to be measured in a methanol solvent, in the absence of aflatoxin added methanol solvent, light source will excite a certain number of photons.During the detection the 
system needs to maintain a stable light source, in order to maintain the baseline within the range of relatively stability. in the experiment the system can also be detected indirectly by the baseline source of stability $[8,9,10]$.

According to the characteristics of the light source required for the experiment, LED lights can be seen from the spectral curve of Figure 1, the wavelength of the light source needs to 370nm, Light source of the cathode has very high sensitivity and quantum efficiency of radiation, so the selected LED lamp model is NSPU510CS .

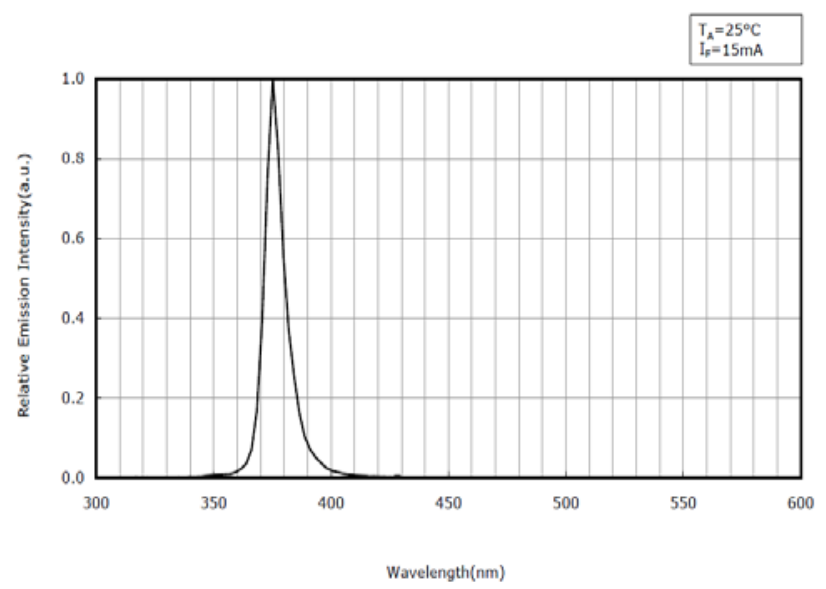

Figure 1 Spectral graph of LED lamp

For the stability controlling of LED light source, it is mainly the use ADN2830 controller. The feedback of lighting source is mainly used S1087 as a main light battery feedback device . ADN2830 provides closed loop feedback control loop for a continuous wave light source, controlling loop provides stable current through the real-time monitoring of the current of LED so as to maintain a constant optical power. the advantage of using this controller can be changed, through a small-scale circuit design, you can adjust the desired optical power.

OP2177 used in the feedback loop of the light source mainly compose of the I / V conversion circuits. The controller supplies power which can light LED, the intensity of the light generated by the LED is converted to a current output after the light battery receiving, through I / V conversion circuit, the voltage feedback to the controller, controllers adjust automatically in accordance with the desired light intensity. Since the current generated by photovoltaic cells reached uA smaller level, so operational amplifier should have high-precision, unity-gain stable, low noise, low input bias current. Light feedback regulation circuit shown in Figure 2.

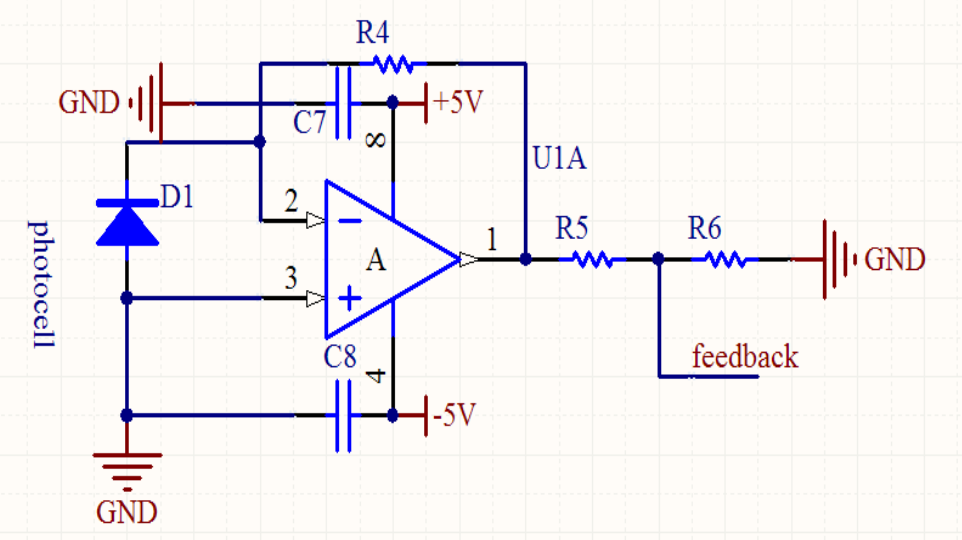

Figure 2 The light intensity feedback circuit diagram

\section{Hardware testing and data analysis}

The main objective of the continuous wave light source hardware design is to obtain a long and 
stable source to provide a suitable light source, the light source is mainly reflected in the stability of voltage, current and optical power data acquisition, The stability of the light source mainly reflects in the long baseline and the actual curve smooth degree of aflatoxin detection.

Verify the stability of the first light source circuit is through the limited acquisition of voltage, current and optical power source. in Table 1 is the voltage, current and optical power data source circuit.

Table 1 Voltage, current and optical power of the source circuit

\begin{tabular}{lccc}
\hline $\begin{array}{c}\text { Collection time/ } \\
\text { min }\end{array}$ & $\begin{array}{c}\text { current / } \\
\mathrm{mA}\end{array}$ & $\begin{array}{c}\text { voltage / } \\
\mathrm{V}\end{array}$ & $\begin{array}{c}\text { optical power / } \\
\mathrm{mW}\end{array}$ \\
\hline 0 & 25.31 & 3.428 & 4.7 \\
30 & 25.32 & 3.425 & 4.8 \\
60 & 25.34 & 3.423 & 4.7 \\
120 & 25.32 & 3.423 & 4.7 \\
\hline
\end{tabular}

As is saw the light source can be more intuitive through operating voltage and operating current through the above data, based on the formula of Data Reference Manual LED optical power is within the rated parameters. seen from the above data LED light will keep long-term stable and efficient.

The design of the continuous wave light source is used in the detection of aflatoxin, aflatoxin is detected by displaying the content of the process of the host computer, it clearly distinguish the stability of the light source. FIG. 3 is a waveform diagram baseline detection .

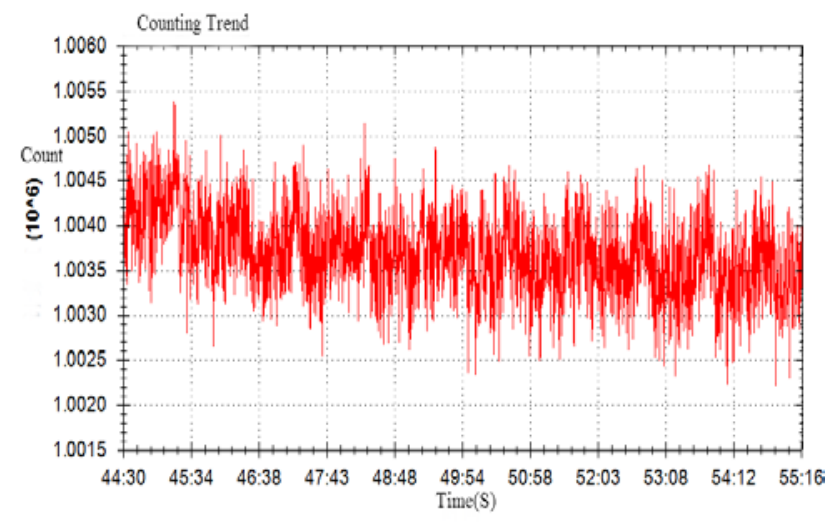

Figure 3 Waveform of the baseline

A graph of the Aflatoxin G1, B1, G2, B2 is in FIG. 4.

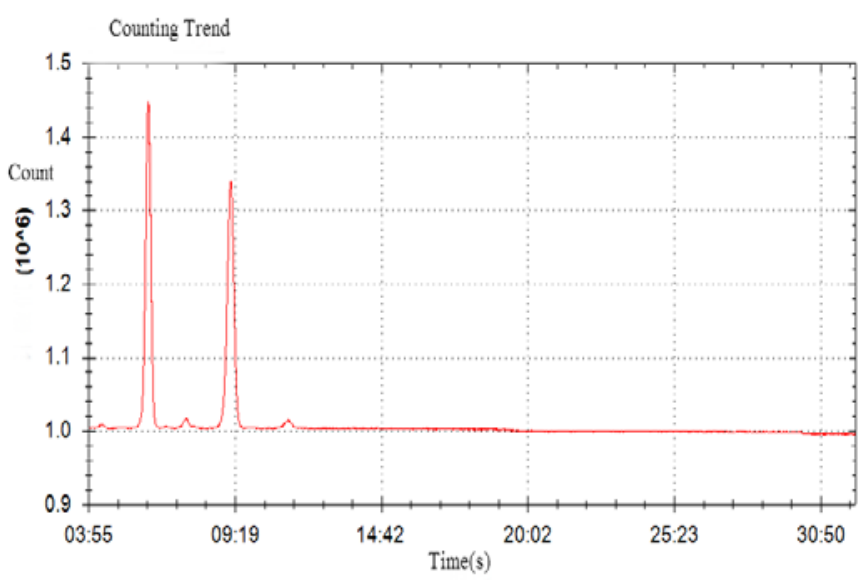

Figure 4 The G1、B1、G2、B2 curve of Aflatoxins 
Figure 3 is a curve about the system stability test before the detection of aflatoxin, it could also be seen as the detection of the stability of light source before the start of the experiment,.Seen from the baseline data of the curve is within the range of 2000, allowed within the error range, it indicates that the light source is maintained at a relatively stable state. after injection baseline detection of aflatoxin appears G1, B1, G2, B2 four peaks, it could clearly distinguish the waveform of each element in the curve, after this curve has remained in a relatively stable state. from the above collected data and testing of the acquired image can effectively illustrate the design of a continuous wave light source could meet the requirements of stability, it can also effectively analyze aflatoxin elemental.

\section{Conclusion}

The design of this system is based on the automatic control of closed loop control system principle, the system can achieve that the real-time power of LED lights automatically adjust, and the optical power ultimately be stable. Through the debugging of the whole hardware and the used in the detection of aflatoxin , analysis of the resulting data is available and the system is stable, it could meet the needs of the experiments. the system can be used in a variety of chemiluminescent detection.

\section{Acknowledgment}

This work was supported by Zhejiang Science and Technology Program (2015C33009), Jiaxing Science and Technology Program (2015AY11008).

\section{References}

[1] Zain M E.Journal of Saudi Chemical Society[J].2011,15(2): 129-144.

[2] Lewis L,Onsongo M,Njapau H.Environ Health Perspect[J].2005,113(12): 1763-1767.

[3] Chen Nan.Introduction the experience in the Aflaloxins testing[J]. Jiangxi Chemical Industry, 2009,4: 9-11.

[4] Mather J, Rainville P D, Spooner N, Evans C A.Bioanalysis[J].2011,3(4): 411-420

[5] Determination of Aflatoxinscontent in Food-Cleanup by Immunoaffinity Chromatography and Determination by High-performance Liquid Chromatography and Fluormeter[S].National Standards of the People's Republic of China.GB/T 18979-2003.

[6] Li Weiyong,Chang Dading,Zeng Yanan.Single - Photon Counting System Research Based on CPLD[J]. Computer \& Digital Engineering,2006,38 (6): 84-88.

[7] Design and implementation of the single photon counter system[J]. Chinese Journal of Scientific Instrument, 2007,28 (4): 28-30.

[8] Shi Yunfei,Jin Yi.Study on the Method for Determination of Aflatoxinin Peanut[J]. Chinese Journal of Health Laboratory Technology, 2012,22 (11): 2584-2585.

[9] Peng Zhibing,Zhang Xuan,Jiang Jianyun.Analysis of Aflatoxin in Grain by Liquid-Liquid Extraction-High Performance Liquid Chromatography[J]. Grain Technology and Economy,2013,38 (1): $26-29$.

[10] Chen Dongdong,Dai Hanxia,Peng Tao. Determination of six aflatoxins in animal liver by photochemical derivative-high performance liquid chromatography combining with immunoaffinity column clean-up[J]. Journal of Food Safety and Quality,2014,5(3):796-800. 